\title{
Silkworm Growth Monitoring Smart Sericulture System based on Internet of Things (IOT) and Image Processing
}

\author{
Nivaashini M. \\ Department of Computer \\ Science \& Engineering \\ Bannari Amman Institute of \\ Technology \\ Sathyamangalam, Erode, \\ India
}

\author{
Soundariya R. S. \\ Department of Computer \\ Science \& Engineering \\ Bannari Amman Institute of \\ Technology \\ Sathyamangalam, Erode, \\ India
}

\author{
Dinesh Kumar A. \\ Department of Information \\ Technology \\ PSG College of Technology \\ Coimbatore, India
}

\begin{abstract}
The art of culturing silkworm for the production of silk is termed as sericulture. Globally, the second biggest manufacturer of silk is India. In India, sericulture acts as the base for financial, social, political and intellectual advancements. Moistness and temperature take part a critical position in the progression of solid silkworms in each stage, particularly in the duration of the improvement of hatchling. Sterilization is one of the basic parameter to be considered for solid and effective silkworm nurturing. The proposed framework introduces an Internet of Things (IoT) empowered Wireless Personal Area Network (WPAN) system in order to deal with a continuous observation of silkworm development in sericulture and picture handling innovation to recognize the phases of silk worm life cycle. The proposed model is employed utilizing Arduino Software and sensors to gauge the environmental circumstances within the arrangement of silkworms according to the prerequisites for each and every stages of silkworm life cycle. The entire model will be fabricated utilizing the Arduino Board stack integrated with moistness and temperature sensors in the company of a camera to catch the photos and to examine it utilizing a picture preparing technique to confirm the condition of sericulture progression.
\end{abstract}

\section{Keywords}

Sericulture, WPAN, IoT, Arduino, Sensors, GSM900 module, Image processing.

\section{INTRODUCTION}

One of the recent trends in technology is Internet of Things (IoT), which made diversity of every object toward detection, activation and transmission via web through perceiving itself by means of an exclusive addressing format; in addition, the objects are connected remotely to make an elegant usage. More than M2M correspondence, Wireless Sensor Networks (WSN), Radio Frequency IDentification (RFID), 2G/3G/4G, and Bluetooth, the performance of IoT is considerably better. Internet of Things is an organization of set of network connections and network systems by means of remarkable features that holds applications in different spaces. In day to day life, every field like urban communities, ventures, agribusiness, transportation, vitality, training, etc are merged with IoT through its own smart knowledge.

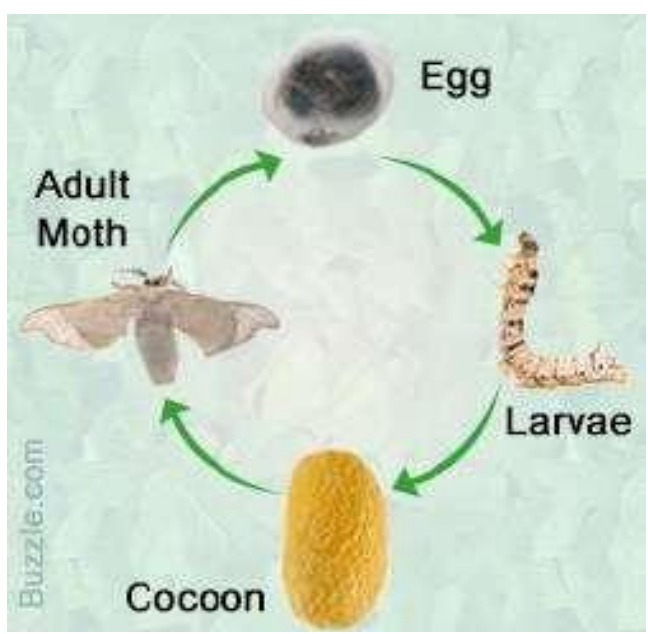

Fig. 1. The lifecycle of the silkworm

Asia is the primary maker of silk with mass generation of it is in China and India, which creates more than $95 \%$ of the aggregate worldwide yield, trailed next to nations like Brazil, Korea, Japan, Italy and USA. Silkworms are nourished exclusively by feeding mulberry plants or potentially by silkworm foodstuffs. The stenophagous insects don't consume or swallow, during the matured stage of the sericulture. The development life cycle of silkworm comprises of four unique stages as shown in the fig. 1, they are egg, hatchling, pupa and moth. The activities in the lifecycle of the silkworm are divided into binary partitions: the agric-based part as well as the trade part. The agric-based part includes binary stages of actions that include mulberry development along with silkworm nurturing. The nurturing of silkworm is separated into binary phases: immature age nurturing in first and second instar. Following that, the middle phase represents the third instar, finally the fourth and fifth instars goes under late age nurturing.

The proposed elegant framework includes shrewd sensor hubs integrated by means of moistness and temperature sensors in order to gather constant precise readings inside the framework surrounded by limited stages. The technique that is used to catch the photos and examine the various conditions of sericulture life cycle is image processing. The proposed system makes use of WPAN (Wireless Personal Area 
Network) for wireless connections along with Arduino Board stack, software, temperature and moistness sensors and a GSM900 sim module to send the ready message if the temperature or the moistness is in dangerous range.

\section{RELATED WORK}

DivyaDarshini.B et al developed an elegant Sericulture model in view of IPv6 over Low Power Wireless Personal Area Network (6LoWPAN) and Image Processing Technique in a way to deal with an ongoing sericulture checking and sanitization activating framework with a consideration of picture handling innovation to distinguish the phases of sericulture process [1].

Sunita et al build up a general auxiliary plan that deals with the necessities of remote sensor hub. The auxiliary plan is not fixed to a specific radio or handling novelty yet rather points of interest how the calculation and communication should be combined together [2]. V.K.Rahmathulla experimented that the cyclic variations in the environmental mechanisms significantly influence the genotypic articulation as phenotypic yield of silkworm harvest that includes shell weight, cocoon mass and shell proportion [5]. The differences in the ecological circumstances are recorded from time to time in order to highlight the need of supervision of moistness and temperature for feasible cocoon generation.

Shakil Ahmad Khan Studied the warmth prerequisite for silkworm larval phase of 27 days to make sure the most favorable ecological conditions in the nurturing room in April and May. Firstly, when silkworm nurturing started on April 20 , it was resolved that the nurturing room should be maintained warm during the larval period [6]. Konstantinos N worked on adaptive filters for color image processing which constitutes a prevailing framework for processing multichannel signals and dealt with the problem of color image filtering by making use of non-parametric methodologies and Bayesian method, as a worldwide perspective that promptly yields and brings together the preceding dissimilar outcomes [10].

\section{SERICULTURE SYSTEM MONITORING}

Silk is the emperor of textile world because of numerous qualities such as sparkling gloss, delicate quality, style, toughness, and tractability. A protein fiber called silk is discharged from the silkworm hatchling in support of cocoon turning. The significance of Silkworm is the generation of silk. The larvae of Silkworm are nourished by means of mulberry plants and it turns their silken cocoons after the fourth shed. The moistness and temperature assumes a key part in each phase of sericulture process, and a great deal of care is additionally should have been taken to maintain a strategic distance from sicknesses. Temperature, moistness and picture process ought to be figured out in fig 2 to get an awesome silk item.

A perfect temperature of $220 \mathrm{C}$ to $280 \mathrm{C}$ and moistness in the middle of $65 \%$ - $85 \%$ must be maintained. In order to maintain the moistness and temperature as per the requirement, a computerized checking based on the IoT activation framework is composed and executed.

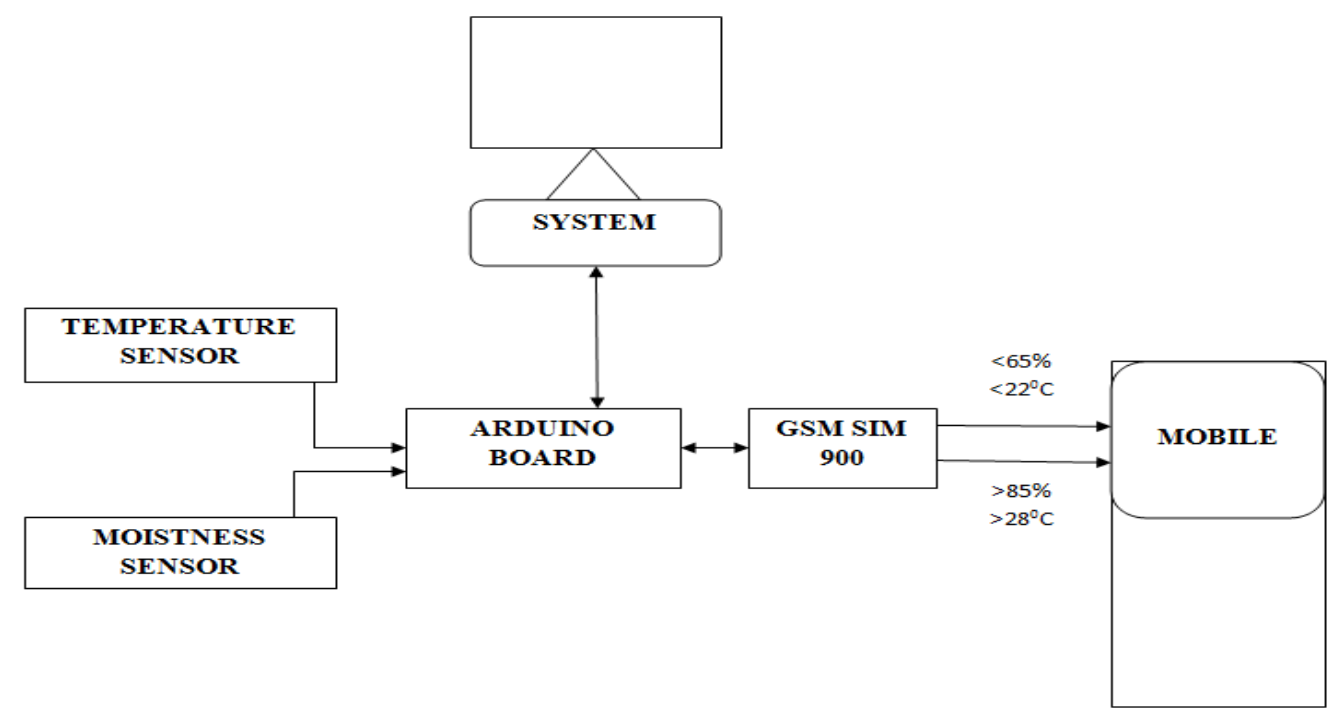

Fig. 2. Systematic Design of the Proposed System

\section{PROTOTYPE IMPLEMENTATION}

Arduino is an open-source platform utilized for the construction of electronic gadgets. Arduino comprises of both a physical programmable circuit board (microcontroller) and a portion of Integrated Development Environment (IDE) that keeps running on the Personal Computer (PC), used to compose and transfer PC code to the physical board. Arduino Uno is a microcontroller board with 6 analog inputs, 14 digital input/output pins, a power jack, USB connection, a reset button and ICSP header. It comprises of all the components that supports the microcontroller.

The structure of DHT11 sensor includes a row of 3-pin package that works from 3.5 to $5.5 \mathrm{~V}$ power supply and determine temperature starting from $0-50^{\circ} \mathrm{C}$ with correctness of $\pm 2{ }^{\circ} \mathrm{C}$ and moistness varying starting from $20-95 \%$ with precision of $\pm 5 \%$. A completely adjusted computerized yield to the two estimations is given by the sensors. It possesses an exclusive single wire convention, consequently, the contact 
among a microcontroller and the sensor is impractical via an immediate edge along with its peripherals. The firmware of the MCU convention must be executed with exact planning by means of sensor.

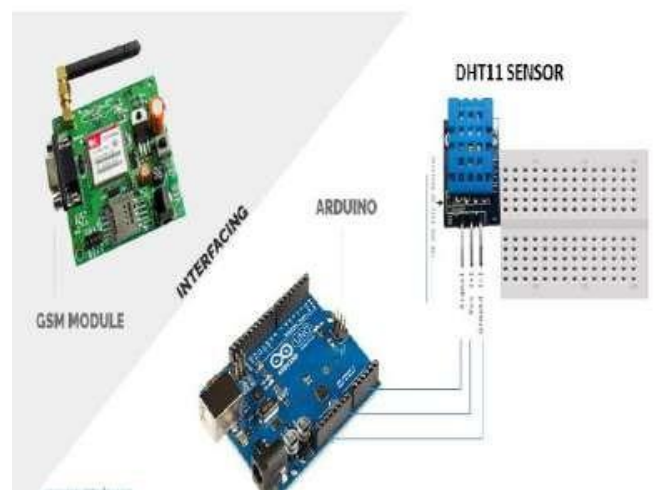

Fig. 3. Ardunio, Sensor, GSM module interface

GSM Sim900A Modem GSM/GPRS Modem- RS232 is constructed by means of Dual Band GSM/GPRS motor SIM900A, operates on frequencies 900/ $1800 \mathrm{MHz}$. GSM Sim900A is accompanied with RS232 interface, which permits connection between the PC and microcontroller with RS232 Chip (MAX232). The baud rate is configurable from 9600-115200 through AT command. The GSM/GPRS Modem is having internal TCP/IP stack to connect with internet via GPRS and it is suitable for SMS, Voice as well as DATA transfers' application in M2M interface. This modem facilitates audio calls, SMS, Read SMS, attend the incoming calls and web through simple AT commands. Normal operational temperature ranges from $-20{ }^{\circ} \mathrm{C}$ to $+55{ }^{\circ} \mathrm{C}$ and Input Voltage is $12 \mathrm{~V}$ DC. The Ardunio, Sensor, GSM module are interfaced in the bread board, using which the temperature and the moistness is sensed.

If the temperature $(<220 \mathrm{c}$ or $>280 \mathrm{c})$ and the moistness $(<65 \%$ or $>85 \%)$ is critical. The system automatically sends the message to the concern person "the temperature is critical with the reading".

\section{IMAGE PROCESSING OF SERICULTURE}

The original captured image of the silkworm is converted into a computerized frame using image processing, further it is used to extract the improved version of the image or some valuable information from the image. To undergo further processing of the image, strategies like Digital and Analog Image processing are utilized to achieve the enhanced version of the original images. Printed versions like printouts and photos can be done using Analog or visual techniques. Handling of the digitalized images is executed using Digital
Image processing in PCs. The raw data gathered through the serial camera is subjected to three stages of processing called pre-processing, enhancement and display, information extraction. For mathematical calculation, perception, and application improvement Matlab is utilized by implementing different implicit algorithms.

Generally, images are incorporated as two dimensional signals in image processing framework while applying effective signal processing techniques. Recognization of the worms' body color change to the light yellowish shows the diverse phases and demonstrates that the worms have attained the cocoon stage.

\section{RESULT ANALYSIS}

The experimental results of monitoring the sericulture farm using Ardunio and GSM module has been described in this section. Fig. 4 represents the SMS received through GSM module about the critical temperature condition in the sericulture frame sensed by the temperature sensor.

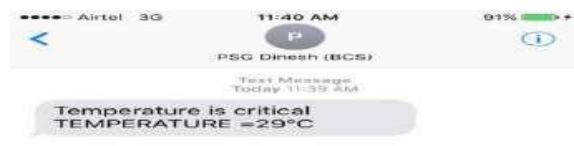

\section{Fig. 4. The real time temperature sensor value}

The growth of the silkworm in four different stages has been monitored using the camera and image processing technique, fig. 5 shows color representation of the silkworm in the dimension of Hue, the dominance of the color change is represented in the dimension of Saturation and the brightness is represented in the dimension of Value. Color position and color purity is the measures used in the color detection process.

Fig. 6 depicts the tonal distribution of the digital image in terms of histogram graphical representation. The graph represents the plots between numbers of pixels to each tonal value. Fig. 7 explains the color change for each dimensional change as mentioned in fig.5. Original color image indicates yellow color; Hue image indicates green color and so on. Based on this color detection process, the normal and abnormal growth of the silkworm can be predicted. 


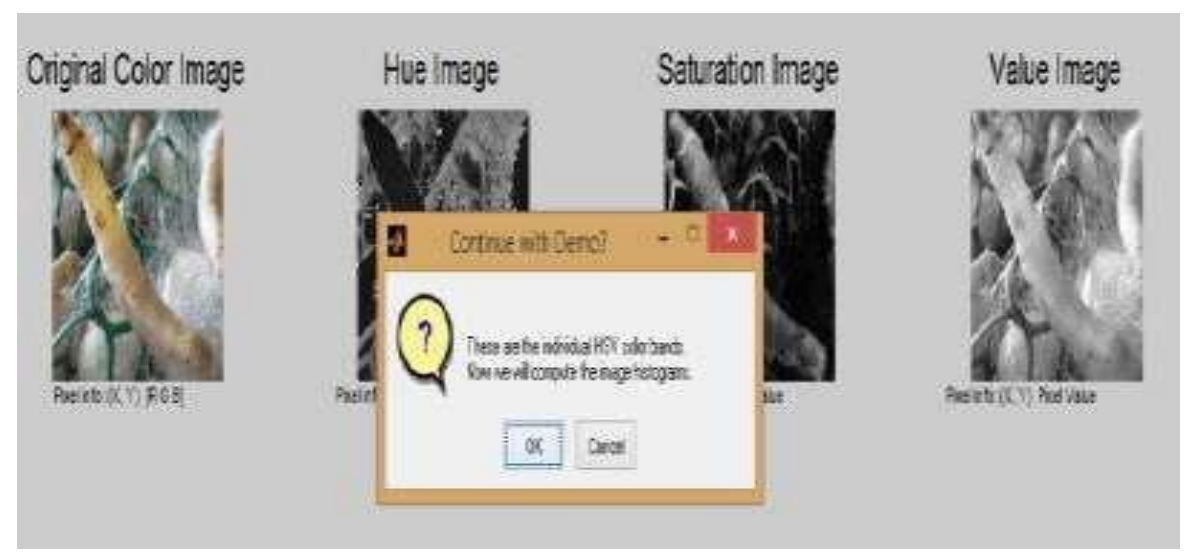

Fig. 5. Image processing (RGB to HSV)

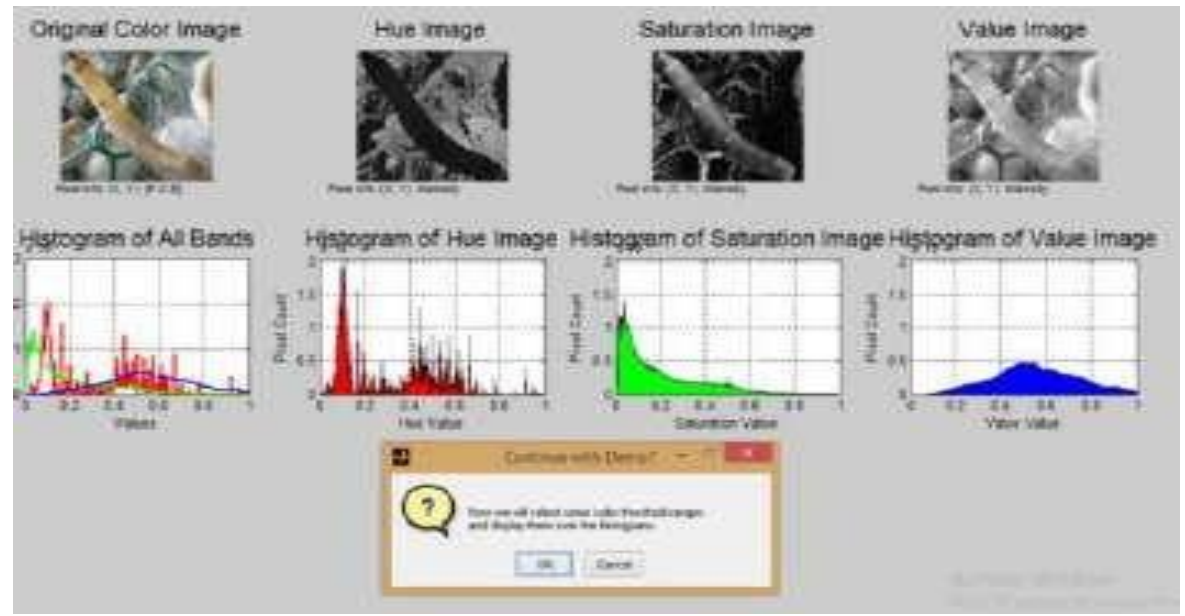

Fig. 6. Transformation of image

Table. 1 explains the value of temperature and moistness for the proper development of the silkworm at each stage of its lifecycle. If the range of temperature and moistness exceeds the tabulated values, then the GSM module is used to indicate the critical temperature and moistness value.

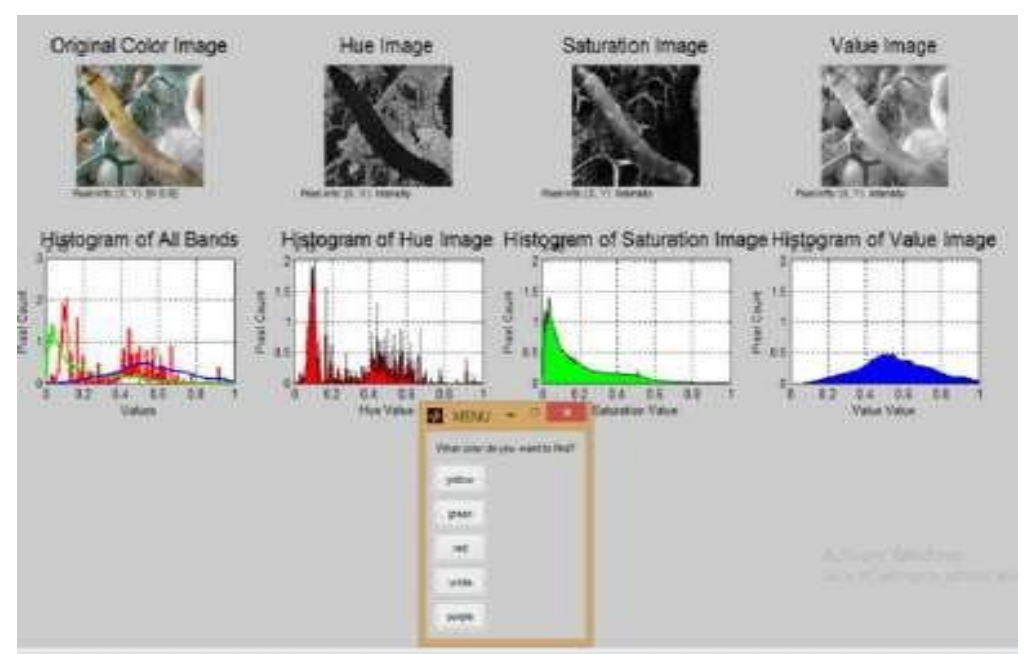

Fig. 7. Image colour detection 
Table 1. Temperature and moistness records for the proper growth of silkworm

\begin{tabular}{|c|c|c|}
\hline Stages & Temperature & Moistness \\
\hline Egg & 240c to 260c & $75 \%-80 \%$ \\
\hline Larvae & $220 \mathrm{c}$ to $280 \mathrm{c}$ & $85 \%$ \\
\hline Cocoon & 240c & $70 \%$ \\
\hline Adult moth & 250c & $80 \%$ \\
\hline
\end{tabular}

\section{CONCLUSION}

This paper introduces an outline of IoT based elegant monitoring and image processing methods. The model will work continuously to monitor the framework and preparatory test demonstrates that actualized model effectively send the message by screening the parameters progressively. Image processing is done in order to identify the continuous condition in total sericulture process and to distinguish different phases in the lifecycle of the silkworm based on the color images. The framework thus executed is cost-effective, adaptable, ease of use and efficient. Future work incorporates the field organization of sericulture for continuous observing over the web. To build the system more elegant and intellectual, sensors other than temperature and moistness can be interfaced with the proposed framework.

\section{REFERENCES}

[1] DivyaDarshini.B,Adarsh.B,Shivayogappa.H.J,Navya. K.N"Automated Smart Sericulture System based on 6LoWPAN and Image Processing Technique" 2016 International Conference on Computer Communication and Informatics (ICCCI -2016), Jan. 07 - 09, 2016, Coimbatore.

[2] Ms.Sunita, Jyoti Malik and Suman Mor, "Comprehensive Study of Applications Sensor Network", International Journal of Advanced Computer Science Research and Software Engineering, Vol 2, Issue 11, Nov 2012.

[3] Jonathan Isaac Chanin and Andrew R. Halloran, "Wireless Sensor Network for Monitoring Applications", a major qualifying project report submitted to the University of Worcester Polytechnic Institute.

[4] Guobao Xu, Weiming Shen and Xianbin Wang, "Applications of Wireless Sensor Networks in Marine Environment Monitoring: A Survey", ISSN 1424-8220, 2014.

[5] V.K.Rahmathulla, "Management of Climatic Factors for Successful Silkworm (Bombyxmori) Crop and Higher Silk Production : A Review", Hindawi Publishing Corporation Psyche, Volume 2012, Article ID 121234, 12 pages doi: $10.1155 / 2012 / 121234$.

[6] Mubashar Hussain, Shakil Ahmad Khan, Muhammad Naeem and M. Farooq Nasir, "Effect of Rearing Temperature and Humidity on Fecundity and Fertility of Silkworm, Bombyxmori L. (Lepidoptera: Bombycidae)" Pakistan J. Zool., vol. 43(5), pp. 979- 985, 2011.

[7] B.R. Patil, K.K. Singh, S.E. Pawar, L. Maarse and J. Otte, "Sericulture: An Alternative Source of Income to Enhance the Livelihoods of Small-scale Farmers and Tribal Communities", Pro-Poor Livestock Policy Initiative A Living from Livestock Research Report, RR Nr. 09-03; July 2009.

[8] P. Levis, T. Clausen, J. Hui, O. Gnawali, and J. Ko, "The Trickle Algorithm", RFC 6206 (Proposed Standard), March 2011.

[9] P. Thubert, "Objective Function Zero for the Routing Protocol for Low-Power and Lossy Networks (RPL)” RFC 6552 (Proposed Standard), March 2012.

[10] Konstantinos N. Plataniotis, DimitriosAndroutsos, Sri Vinayagamoorthy, and Anastasios N. Venetsanopoulos, "Color Image Processing Using Adaptive Multichannel Filters", IEEE Transactions on Image Processing, VOL. 6, NO. 7, July 1997.

[11] RicardoL.deQueiroz, "Processing JPEG-Compressed Images and Documents", IEEE Transactions on Image Processing, VOL. 7, NO.12-December1998. 